\title{
Microbiology Susceptibility Sequence Number
}

National Cancer Institute

\section{Source}

National Cancer Institute. Microbiology Susceptibility Sequence Number. NCI Thesaurus.

Code C87941.

A number that identifies the ordering relations (in time, space, etc.) in a set of microbiology susceptibility data. 